\title{
STRONG CONVERGENCE OF APPROXIMATING FIXED POINTS FOR NONEXPANSIVE NONSELF-MAPPINGS IN BANACH SPACES
}

\author{
Jong Soo Jung and Tae Hwa Kim
}

\begin{abstract}
Let $E$ be a reflexıve Banach space with a uniformly Gâteaux differentiable norm, $C$ a nonempty closed convex subset of $E$, and $T C \rightarrow E$ a nonexpansive mapping satisfying the inwardness condition. Assume that every weakly compact convex subset of $E$ has the fixed point property. For $u \in C$ and $t \in(0,1)$, let $x_{t}$ be a unique fixed point of a contraction $G_{t} \quad C \rightarrow E$, defined by $G_{t} x=t T x+(1-t) u, x \in C$. It is proved that if $\left\{x_{t}\right\}$ is bounded, then the strong $\lim _{t \rightarrow 1} x_{t}$ exists and belongs to the fixed point set of $T$ Furthermore, the strong convergence of other two schemes involving the sunny nonexpansive retraction is also given in a reflexive and strictly convex Banach space with a uniformly Gâteaux differentiable norm.
\end{abstract}

\section{Introduction}

Let $C$ be a nonempty closed convex subset of a Banach space $E$, and let $T$ : $C \rightarrow E$ be a nonexpansive mapping (i.e., $\|T x-T y\| \leq\|x-y\|$ for all $x, y \in C$ ). Given a $u \in C$ and a $t \in(0,1)$, we can define a contraction $G_{t}: C \rightarrow E$ by

$$
G_{t} x=t T x+(1-t) u, \quad x \in C .
$$

If $T$ is a self-mapping (i.e., $T(C) \subset C$ ), then $G_{t}$ maps $C$ into itself, and hence, by Banach's contraction principle, $G_{t}$ has a unique fixed point $x_{t}$ in $C$, that is, we have

$$
x_{t}=t T x_{t}+(1-t) u \text {. }
$$

(Such a sequence $\left\{x_{t}\right\}$ is said to be an approximating fixed point of $T$ since it possesses the property that if $\left\{x_{t}\right\}$ is bounded, then $\lim _{t \rightarrow 1}\left\|T x_{t}-x_{t}\right\|=0$.) The strong convergence of $\left\{x_{t}\right\}$ as $t \rightarrow 1$ for a self-mapping $T$ of a bounded $C$ was proved in a Hilbert space independently by Browder [2] and Halpern [10] and in

1991 Mathematics Subject Classification. Prımary 47H09; Secondary 47H10.

Key words and phrases. Nonexpansive mapping, fixed points, boundary conditions, weak inwardness, Banach limits, sunny and nonexpansive retraction.

The authors were supported in part by the Basic Science Research Institute Program, Ministry of Education, Korea, 1997, Project No. BSRI-97-1405 and Project No. BSRI-97-1440, respectively.

Received June 16, 1997; revised April 23, 1998. 
a uniformly smooth Banach space by Reich [18]. Thereafter, Singh and Watson [21] extended the result of Browder and Halpern to a nonexpansive nonselfmapping $T$ satisfying Rothe's boundary condition: $T(\partial C) \subset C$ (here $\partial C$ denotes the boundary of $C$ ).

Recently, $\mathrm{Xu}$ and $\mathrm{Yin}$ [27] proved that if $C$ is a nonempty closed convex (not necessarily bounded) subset of Hilbert space $H$, if $T: C \rightarrow H$ is a nonexpansive nonself-mapping, and if $\left\{x_{t}\right\}$ is the sequence defined by (2) which is bounded, then $\left\{x_{t}\right\}$ converges strongly as $t \rightarrow 1$ to a fixed point of $T$. They also studied other two schemes involving the nearest point projection $P$ from $H$ onto $C$, which were introduced by Marino and Trombetta [10]. Jung and Kim [11] extended $\mathrm{Xu}$ and Yin's results [27] to a uniformly convex Banach space with a uniformly Gâteaux differentiable norm with the additional condition upon $C$. Kim and Takahashi [12] also generalized $\mathrm{Xu}$ and Yin's results [27] to a smooth and reflexive Banach space with a weakly sequentially continuous duality mapping.

Very recently, $\mathrm{Xu}$ [26] showed that if $E$ is a uniformly smooth Banach space, if $C$ is a nonempty closed convex subset of $E$, and if $T: C \rightarrow E$ is a nonexpansive nonself-mapping with a fixed point, which satisfies the inwardness condition, then the sequence $\left\{x_{t}\right\}$ defined by (2) converges strongly as $t \rightarrow 1$ to a fixed point of $T$. He also gave the strong convergence theorem in a uniformly convex and uniformly smooth Banach space with the weak inwardness condition upon the mapping $T$.

In this paper, we establish the strong convergence of $\left\{x_{t}\right\}$ defined by (2) for a nonexpansive nonself-mapping $T$ in a reflexive Banach space with a uniformly Gâteaux differentiable norm. We also prove the strong convergence of other two schemes studied in $[12,13,27]$ in a reflexive and strictly convex Banach space with a uniformly Gâteaux differentiable norm. Our results extend and improve the results in $[18,26,27]$.

\section{Preliminaries}

Let $E$ be a real Banach space with norm $\|\cdot\|$ and let $E^{*}$ be its dual. The value of $x^{*} \in E^{*}$ at $x \in E$ will be denoted by $\left(x, x^{*}\right)$.

A Banach space $E$ is called strictly convex if its unit sphere $U=\{x \in E$ : $\|x\|=1\}$ does not contain any linear segment. For every $\varepsilon$ with $0 \leq \varepsilon \leq 2$, the modulus $\delta(\varepsilon)$ of convexity of $E$ is defined by

$$
\delta(\varepsilon)=\inf \left\{1-\left\|\frac{x+y}{2}\right\|:\|x\| \leq 1,\|y\| \leq 1,\|x-y\| \geq \varepsilon\right\} .
$$

$E$ is said to be uniformly convex if $\delta(\varepsilon)>0$ for every $\varepsilon>0$. If $E$ is uniformly convex, then $E$ is reflexive and strictly convex.

The norm of $E$ is said to be Gâteaux differentiable (and $E$ is said to be smooth) if

$$
\lim _{t \rightarrow 0} \frac{\|x+t y\|-\|x\|}{t}
$$


exists for each $x, y$ in its unit sphere $U=\{x \in E:\|x\|=1\}$. It is said to be uniformly Gâteaux differentiable if, for each $y \in U$, this limit is attained uniformly for $x \in U$. Finally, the norm is said to be uniformly Fréchet differentiable (and $E$ is said to be uniformly smooth) if the limit in (3) is attained uniformly for $(x, y) \in$ $U \times U$. Since the dual $E^{*}$ of $E$ is uniformly convex if and only if the norm of $E$ is uniformly Fréchet differentiable, every Banach space with a uniformly convex dual is reflexive and has a uniformly Gâteaux differentiable norm. The converse implication is false. A discussion of these and related concepts may be found in [5].

The (normalized) duality mapping $J$ from $E$ into the family of nonempty (by Hahn-Banach theorem) weak-star compact subsets of its dual $E^{*}$ is defined by

$$
J(x)=\left\{f \in E^{*}:(x, f)=\|x\|^{2}=\|f\|^{2}\right\} .
$$

for each $x \in E$. It is single valued if and only if $E$ is smooth. It is also wellknown that if $E$ has a uniformly Gâteaux differentiable norm, $J$ is uniformly continuous on bounded subsets of $E$ from the strong topology of $E$ to the weakstar topology of $E^{*}$. This fact is explicitly proved in Lemma 2.2 of [19] (see also $[4,6,7])$.

Let $\mu$ be a mean on positive integers $N$, i.e., a continuous linear functional on $\ell^{\infty}$ satisfying $\|\mu\|=1=\mu(1)$. Then we known that $\mu$ is a mean on $N$ if and only if

$$
\inf \left\{a_{n}: n \in \boldsymbol{N}\right\} \leq \mu(a) \leq \sup \left\{a_{n}: n \in \boldsymbol{N}\right\}
$$

for every $a=\left(a_{1}, a_{2}, \ldots\right) \in \ell^{\infty}$. According to time and circumstances, we use $\mu_{n}\left(a_{n}\right)$ instead of $\mu(a)$. A mean $\mu$ on $N$ is called a Banach limit if

$$
\mu_{n}\left(a_{n}\right)=\mu_{n}\left(a_{n+1}\right)
$$

for every $a=\left(a_{1}, a_{2}, \ldots\right) \in \ell^{\infty}$. Using the Hahn-Banach theorem, we can prove the existence of a Banach limit. We know that if $\mu$ is a Banach limit, then

$$
\liminf _{n \rightarrow \infty} a_{n} \leq \mu_{n}\left(a_{n}\right) \leq \limsup _{n \rightarrow \infty} a_{n}
$$

for every $a=\left(a_{1}, a_{2}, \ldots\right) \in \ell^{\infty}$. Let $\left\{x_{n}\right\}$ be a bounded sequence in $E$. Then we can define the real valued continuous convex function $\phi$ on $E$ by

for each $z \in E$.

$$
\phi(z)=\mu_{n}\left\|x_{n}-z\right\|^{2}
$$

The following lemma which was given in $[8,9,23]$ is, in fact, a variant of Lemma 1.3 in [17] (cf. [20, p. 171]).

Lemma 1. Let $C$ be a nonempty closed convex subset of a Banach space $E$ with a uniformly Gâteaux differentiable norm and let $\left\{x_{n}\right\}$ be a bounded sequence in $E$. Let $\mu$ be a Banach limit and $u \in C$. Then

$$
\mu_{n}\left\|x_{n}-u\right\|^{2}=\min _{y \in C} \mu_{n}\left\|x_{n}-y\right\|^{2}
$$


if and only if

$$
\mu_{n}\left(x-u, J\left(x_{n}-u\right)\right) \leq 0
$$

for all $x \in C$.

Let $I_{C}(x)$ be the inward set of a closed convex subset $C$ of $E$ at $x$ given by

$$
I_{C}(x)=\{z \in E: z=x+\lambda(y-x) \text { for some } y \in C, \lambda \geq 0\} .
$$

A nonself-mapping $T: C \rightarrow E$ is said to satisfy the inwardness condition if $T x \in$ $I_{C}(x)$ for all $x \in C . \quad T$ is also said to satisfy the weak inwardness condition if $T x \in \operatorname{cl} I_{C}(x)$ for all $x \in C$, where $\operatorname{cl} I_{C}(x)$ is the closure of $I_{C}(x)$ in norm topology.

Recall that a closed convex subset $C$ of $E$ is said to have the fixed point property for nonexpansive self-mappings (FPP for short) if every nonexpansive mapping $T: C \rightarrow C$ has a fixed point, that is, there is a point $p \in C$ such that $T p=p$. It is well-known that every bounded closed convex subset of a uniformly convex Banach space has the FPP (cf. [7, p. 22]).

Finally, let $C$ be a nonempty closed convex subset of $E$. A mapping $Q$ of $C$ into $C$ is said to be a retraction if $Q^{2}=Q$. If a mapping $Q$ of $C$ into $C$ is a retraction, then $Q z=z$ for every $z \in R(Q)$, where $R(Q)$ is the range of $Q$. Let $Q$ be a retraction of $E$ onto a closed subset $C$ of $E$. $Q$ is said to be sunny if each point on the ray $\{Q x+t(x-Q x): t>0\}$ is mapped by $Q$ back onto $Q x$, in other words,

$$
Q(Q x+t(x-Q x))=Q x
$$

for all $t \geq 0$ and $x \in E$. If there exists a retraction $Q: E \rightarrow C$ which is both sunny and nonexpansive, then $C$ is said to be a sunny nonexpansive retract. Sunny nonexpansive retracts appear in $[16,17]$.

The following lemma is well-known (cf. [7, p. 48; 14, p. 65]).

Lemma 2. Let $C$ be a closed convex subset of a smooth Banach space $E$ and let $Q: E \rightarrow C$ be a retraction. Then the following the equivalent:

(a) $(x-Q x, J(y-Q x)) \leq 0$ for all $x \in E$ and $y \in C$;

(b) $\|Q z-Q w\|^{2} \leq(z-w, J(Q z-Q w))$ for all $z$ and $w$ in $E$;

(c) $Q$ is both sunny and nonexpansive.

\section{Main results}

In this section, we study the strong convergence of $\left\{x_{t}\right\}$ defined by (2) in a reflexive Banach space with a uniformly Gâteaux differentiable norm.

Now, we state and prove the first main result.

THEOREM 1. Let $E$ be a reflexive Banach space with a uniformly Gâteaux differentiable norm, $C$ a nonempty closed convex subset of $E$, and $T: C \rightarrow E$ a nonexpansive nonself-mapping satisfying the inwardness condition. Assume that 
every weakly compact convex subset of $E$ has the FPP. Suppose that for each $u \in C$ and $t \in(0,1)$, the contraction $G_{t}$ defined by (1) has a (unique) fixed point $x_{t} \in C$. Then $T$ has a fixed point if and only if $\left\{x_{t}\right\}$ remains bounded as $t \rightarrow 1$ and in this case, $\left\{x_{t}\right\}$ converges strongly as $t \rightarrow 1$ to a fixed point to $T$.

Proof. If the fixed point set $F(T)$ of $T$ is nonempty, then $\left\{x_{t}\right\}$ is bounded. In fact, we have $\left\|x_{t}-v\right\| \leq\|u-v\|$ for all $t \in(0,1)$ and $v \in F(T)$.

Suppose conversely that $\left\{x_{t}\right\}$ remains bounded as $t \rightarrow 1$. We now show that $F(T)$ is nonempty and that $\left\{x_{t}\right\}$ converges strongly as $t \rightarrow 1$ to a fixed point of $T$. To this end, we follow ideas of [22] and [23]. Let $t_{n} \rightarrow 1$ and $x_{n}=$ $x t_{n}$. Define $\phi: E \rightarrow[0, \infty)$ by $\phi(z)=\mu_{n}\left\|x_{n}-z\right\|^{2}$. Since $\phi$ is continuous and convex, $\phi(z) \rightarrow \infty$ as $\|z\| \rightarrow \infty$, and $E$ is reflexive, $\phi$ attains its infimum over $C$ (cf. [1, p. 79]). Let $z \in C$ be such that

$$
\mu_{n}\left\|x_{n}-z\right\|^{2}=\min _{y \in C} \mu_{n}\left\|x_{n}-y\right\|^{2}
$$

and let

$$
M=\left\{x \in C: \mu_{n}\left\|x_{n}-x\right\|^{2}=\min _{y \in C} \mu_{n}\left\|x_{n}-y\right\|^{2}\right\} .
$$

Then $M$ is a nonempty bounded closed convex subset of $C$. Since

$$
\left\|x_{t}-T x_{t}\right\|=(1-t)\left\|T x_{t}-x\right\| \rightarrow 0 \text { as } t \rightarrow 1,
$$

we have for $x \in C$

$$
\begin{aligned}
\phi(T x)=\mu_{n}\left\|x_{n}-T_{x}\right\|^{2} & =\mu_{n}\left\|T x_{n}-T x\right\|^{2} \\
& \leq \mu_{n}\left\|x_{n}-x\right\|^{2}=\phi(x) .
\end{aligned}
$$

Now we prove that the inwardness condition of $T$ on $C$ implies the inwardness condition of $T$ on $M$; that is,

$$
T x \in I_{M}(x) \text { for } x \in M \text {. }
$$

In fact, let $x \in M$. The inwardness condition of $T$ on $C$ implies that $T x=$ $x+\lambda(y-x)$ for some $y \in C$ and $\lambda \geq 0$. If $\lambda \leq 1$, then $T x \in C$ by convexity of $C$. From (5), it follows that $T x \in M \subset I_{M}(x)$ and (6) is verified. Assume $\lambda>1$, we can write $y$ in the form $y=r T x+(1-r) x$, where $r=\lambda^{-1} \in(0,1)$. By convexity of $f$ and (5), we obtain

$$
\phi(y) \leq r \phi(T x)+(1-r) \phi(x) \leq \phi(x) \text { for } x \in M .
$$

This implies that $y \in M$ and therefore $T x=x+\lambda(y-x)$ belongs to $I_{M}(x)$ for $x \in M$ and (6) is proved. Thus it follows from Theorem 16.1 of Goebel and Reich [7] that $T$ has a fixed point $z \in M$, that is, $F(T)$ is nonempty. On the 
other hand, for $v \in F(T)$, we have

$$
\begin{aligned}
\left(x_{n}-T x_{n}, J\left(x_{n}-v\right)\right. & =\left(x_{n}-T v+T v-T x_{n}, J\left(x_{n}-v\right)\right) \\
& =\left\|x_{n}-T v\right\|^{2}-\left(T x_{n}-T v, J\left(x_{n}-v\right)\right) \\
& \geq\left\|x_{n}-T v\right\|^{2}-\left\|T x_{n}-T v\right\|\left\|x_{n}-v\right\| \\
& \geq\left\|x_{n}-T v\right\|^{2}-\left\|x_{n}-T v\right\|^{2}=0
\end{aligned}
$$

for all $n$. Since $x_{n}-T x_{n}=\left(1-t_{n}\right)\left(u-T x_{n}\right)$, we get from the above inequality

$$
\begin{aligned}
0 & \leq\left(x_{n}-T x_{n}, J\left(x_{n}-v\right)\right) \\
& =\left(1-t_{n}\right)\left(u-T x_{n}, J\left(x_{n}-v\right)\right)
\end{aligned}
$$

for all $v \in F(T)$ and all $n$. Thus from (4) and (7), we obtain

$$
\mu_{n}\left(x_{n}-u, J\left(x_{n}-v\right)\right) \leq 0
$$

for $v \in F(T)$. From Lemma 1, it follows that

$$
\mu_{n}\left(x-z, J\left(x_{n}-z\right)\right) \leq 0
$$

for all $x \in C$. In particular, we have

$$
\mu_{n}\left(u-z, J\left(x_{n}-z\right)\right) \leq 0 .
$$

Combining (8) and (9), we get

$$
\mu_{n}\left(x_{n}-z, J\left(x_{n}-z\right)\right)=\mu_{n}\left\|x_{n}-z\right\|^{2} \leq 0 .
$$

Therefore, there is a subsequence $\left\{x_{n_{j}}\right\}$ of $\left\{x_{n}\right\}$ which converges strongly to $z$. To complete the proof, suppose that there is another subsequence $\left\{x_{n}\right\}$ of $\left\{x_{n}\right\}$ which converges strongly to (say) $y$. Then $y$ is a fixed point of $T$ by (4). It follows from (8) that

$$
(z-u, J(z-y)) \leq 0
$$

and

$$
(y-u, J(y-z)) \leq 0 .
$$

Adding these two inequalities yields

$$
(z-y, J(z-y))=\|z-y\|^{2} \leq 0
$$

and thus $z=y$. This prove the strong convergence of $\left\{x_{t}\right\}$ to $z$.

COROLlaRY 1 [26]. Let $E$ be a uniformly smooth Banach space, $C$ a nonempty closed convex subset of $E$, and $T: C \rightarrow E$ a nonexpansive nonselfmapping satisfying the inwardness condition. Suppose that for each $u \in C$ and $t \in$ $(0,1)$, the contraction $G_{t}$ defined by (1) has a (unique) fixed point $x_{t} \in C$. Then $T$ has a fixed point if and only if $\left\{x_{t}\right\}$ remains bounded as $t \rightarrow 1$ and in this case, $\left\{x_{t}\right\}$ converges strongly as $t \rightarrow 1$ to a fixed point of $T$. 
For the second main result, we need the following result which was essentially proved by Takahashi and Jeong [24] and here present the brief proof for the sake of completeness.

LeMma 3. Let $E$ be a uniformly convex Banach space, $C$ a nonempty closed convex subset of $E$, and $\left\{x_{n}\right\}$ a bounded sequence of $E$. Then the set

$$
M=\left\{u \in C: \mu_{n}\left\|x_{n}-u\right\|^{2}=\min _{z \in C} \mu_{n}\left\|x_{n}-z\right\|^{2}\right\}
$$

consists of one point.

Proof. Let $\phi(z)=\mu_{n}\left\|x_{n}-z\right\|^{2}$ for each $z \in E$ and $r=\inf \{\phi(z): z \in C\}$. Then, since the function $\phi$ on $C$ is convex and continuous, $\phi(z) \rightarrow \infty$ as $\|z\| \rightarrow \infty$, and $E$ is reflexive, it follows from [1, p. 79] that there exists $u \in C$ with $\phi(u)=r$. Therefore $M$ is nonempty. By Theorem 2 of [25], $\|\cdot\|^{2}$ is uniformly convex on any bounded subset of $E$; especially, we have a continuous increasing function $g=g_{r}:[0, \infty) \rightarrow[0, \infty)$, with $g(0)=0$, such that

$$
\|\lambda x+(1-\lambda) y\|^{2} \leq \lambda\|x\|^{2}+(1-\lambda)\|y\|^{2}-\lambda(1-\lambda) g(\|x-y\|), \quad 0 \leq \lambda \leq 1, \quad x, y \in B_{r},
$$

where $B_{r}$ is the closed ball centered at 0 and with radius $r$ that is big enough so that $B_{r}$ contains $\left\{x_{n}\right\}$. It follows that

$$
\phi(\lambda x+(1-\lambda) y) \leq \lambda \phi(x)+(1-\lambda) \phi(y)-\lambda(1-\lambda) g(\|x-y\|), \quad 0 \leq \lambda \leq 1, \quad x, y \in B_{r} .
$$

This implies that $\phi$ is a strictly convex function on $E$. Thus the minimum point $u$ of $\phi$ is unique, that is, $M$ consists of one point.

THEOREM 2. Let $E$ be a uniformly convex Banach space with a uniformly Gâteaux differentiable norm, $C$ a nonempty closed convex subset of $E$, and $T$ : $C \rightarrow E$ a nonexpansive nonself-mapping satisfying the weak inwardness condition. Suppose that for each $u \in C$ and $t \in(0,1)$, The contraction $G_{t}$ defined by (1) has a (unique) fixed point $x_{t} \in C$. If the fixed point set $F(T)$ of $T$ is nonempty, then $\left\{x_{t}\right\}$ converges strongly as $t \rightarrow 1$ to a fixed point of $T$.

Proof. Let $w \in F(T)$. As in proof of Theorem 1, we have $\left\|x_{t}-w\right\| \leq$ $\|u-w\|$ for all $t \in(0,1)$ and hence $\left\{x_{t}\right\}$ is bounded. We now show that $\left\{x_{t}\right\}$ converges strongly as $t \rightarrow 1$ to a fixed point of $T$. To this end, let $t_{n} \rightarrow 1$ and $x_{n}=x_{t_{n}}$. As in the proof of Theorem 1, we define the same function $\phi$ : $E \rightarrow[0, \infty)$ by $\phi(z)=\mu_{n}\left\|x_{n}-z\right\|^{2}$ and let

$$
M=\left\{x \in C: \mu_{n}\left\|x_{n}-x\right\|^{2}=\min _{y \in C} \mu_{n}\left\|x_{n}-y\right\|^{2}\right\} .
$$

Then, by Lemma 3, we know that $M$ consists of one point, say $z$. We must show that this $z$ is a fixed point of $T$. Since $T$ satisfies the weak inwardness 
condition, there are some $v_{n} \in C$ and $\lambda_{n} \geq 0$ such that

$$
w_{n}:=z+\lambda_{n}\left(v_{n}-z\right) \rightarrow T z \text { strongly. }
$$

If $\lambda_{n} \leq 1$ for infinitely many $n$ and these $n$, then we have $w_{n} \in C$ and hence $T z \in$ $C$. We have $T z=z$ by (5). So, we may assume $\lambda_{n}>1$ for all sufficiently large $n$. We then write

$$
v_{n}=r_{n} w_{n}+\left(1-r_{n}\right) z
$$

where $r_{n}=\lambda_{n}^{-1}$. Suppose $r_{n} \rightarrow 1$. Then $v_{n} \rightarrow T z$ and hence $T z \in C$. By (5), we have $T z=z$. So, without loss of generality, we may assume $r_{n} \leq a<1$. By Theorem 2 of [25], $\|\cdot\|^{2}$ is uniformly convex on any bounded subset of $E$; especially, we have a continuous increasing function $g=g_{r}:[0, \infty) \rightarrow[0, \infty)$, with $g(0)=0$, such that

$\|\lambda x+(1-\lambda) y\|^{2} \leq \lambda\|x\|^{2}+(1-\lambda)\|y\|^{2}-\lambda(1-\lambda) g(\|x-y\|), \quad 0 \leq \lambda \leq 1, \quad x, y \in B_{r}$,

where $B_{r}$ is the closed ball centered at 0 and with radius $r$ such that $B_{r}$ contains $z$ and $\left\{w_{n}\right\}$. It follows that

$$
\phi(\lambda x+(1-\lambda) y) \leq \lambda \phi(x)+(1-\lambda) \phi(y)-\lambda(1-\lambda) g(\|x-y\|) \quad 0 \leq \lambda \leq 1, \quad x, y \in B_{r} .
$$

Noting $v_{n} \in C$, we derive that

$$
\begin{aligned}
\phi(z) & \leq \phi\left(v_{n}\right) \\
& \leq r_{n} \phi\left(w_{n}\right)+\left(1-r_{n}\right) \phi(z)-r_{n}\left(1-r_{n}\right) g\left(\left\|w_{n}-z\right\|\right)
\end{aligned}
$$

and hence

$$
(1-a) g\left(\left\|w_{n}-z\right\|\right) \leq\left(1-r_{n}\right) g\left(\left\|w_{n}-z\right\|\right) .
$$

Taking limit as $n \rightarrow \infty$, we obtain

$$
g(\|T z-z\|) \leq \phi(T z)-\phi(z) \leq 0
$$

by (5). Therefore, $T z=z$, that is, $z$ is a fixed point of $T$. The proof of the strong convergence of $\left\{x_{t}\right\}$ to $z$ is the same as given in the proof of Theorem 1 .

Remark 1. (1) Theorem 1 generalizes $\mathrm{Xu}$ and Yin's result [27, Theorem 1] to a Banach space setting.

(2) Corollary 1 extends Reich's result [18] to nonself-mappings.

(3) Theorem 2 also improves slightly Theorem 2 in [26].

(4) To guarantee the existence of a fixed point of the contraction $G_{t}$ defined by (1), the weak inwardness condition upon the mapping $T$ is used. In fact, it is well-known (cf. [7, 15]) that if $C$, a bounded closed convex subset of a Banach space $E$, has the FPP and a nonexpansive $T: C \rightarrow E$ is weakly inward, then the contraction $G_{t}$ has a fixed point for every $t \in(0,1)$. Hence we have the following corollary. 
Corollary 2. Let E, C, T be as in Theorem 2. Suppose in addition that $C$ is bounded. Then for each $u \in C$, the sequence $\left\{x_{t}\right\}$ defined by (2) converges strongly as $t \rightarrow 1$ to a fixed point of $T$. setting.

Remark 2. (1) Corollary 2 generalizes Corollary 1 in [27] to a Banach space

(2) Since Rothe's boundary condition: $T(\partial C) \subset C$ implies the weak inwardness condition, Corollary 2 also improves upon Theorem in [21].

Next, we denote by $Q$ the sunny and nonexpansive retraction of $E$ onto $C$. Now let $T: C \rightarrow E$ be nonexpansive and let $u \in C$ be fixed. Following Marino and Trombetta [10], we define the contraction $U_{t}$ from $C$ into itself by

$$
U_{t} x=t Q T(x)+(1-t) u, \quad x \in C
$$

and

$$
R_{t} x=Q(t T x+(1-t) u), \quad x \in C .
$$

Then Banach's contraction principle yields a unique point $x_{t}$ (resp. $\left.y_{t}\right) \in C$ that is fixed by $U_{t}\left(\operatorname{resp} . R_{t}\right)$, that is, we have

$$
x_{t}=t Q T\left(x_{t}\right)+(1-t) u
$$

and

$$
y_{t}=Q\left(t T y_{t}+(1-t) u\right) .
$$

The following lemma is well-known (cf. [1, p. 79; 7, p. 12]).

LEMma 4. Let $C$ be a closed convex of a reflexive and strictly convex Banach space $E$. Then $C^{o}=\{x \in C:\|x\|=\inf \{\|y\|: y \in C\}\}$ is a singleton.

THEOREM 3. Let $E$ be a reflexive and strictly convex Banach space with a uniformly Gâteaux differentiable norm, $C$ a nonempty closed convex subset of $E$, and $T: C \rightarrow E$ a nonexpansive nonself-mapping satisfying the weak inwardness condition. Suppose that $C$ is a sunny nonexpansive retract of $E$, and that for some $u \in C$ and each $t \in(0,1), x_{t}$ is a (unique) fixed point of the contraction $U_{t}$ defined by (10), where $Q$ is a sunny nonexpansive retraction of $E$ onto $C$. If the fixed point set of $T$ is nonempty, then $\left\{x_{t}\right\}$ converges strongly as $t \rightarrow 1$ to a fixed point of $T$.

Proof. We follow an idea of [22]. Let $w \in F(T)$. Then it is easily seen that $\left\|x_{t}-w\right\| \leq\|u-w\|$ for all $t \in(0,1)$ and hence $\left\{x_{t}\right\}$ is bounded. As in the proof of Theorem 1 , we define the same function $\phi \cdot C \rightarrow[0, \infty)$ by $\phi(z)=$ $\mu_{n}\left\|x_{n}-z\right\|^{2}$ and let

$$
M=\left\{x \in C: \mu_{n}\left\|x_{n}-x\right\|^{2}=\min _{y \in C} \mu_{n}\left\|x_{n}-y\right\|^{2}\right\} .
$$


Then $M$ is invariant under $Q T$. In fact, since

$$
\left\|x_{t}-Q T x_{t}\right\|=(1-t)\left\|Q T x_{t}-x\right\| \rightarrow 0 \quad \text { as } t \rightarrow 1,
$$

we have for $x \in M$

$$
\begin{aligned}
\phi(Q T x)=\mu_{n}\left\|x_{n}-Q T x\right\|^{2} & =\mu_{n}\left\|Q T x_{n}-Q T x\right\|^{2} \\
& \leq \mu_{n}\left\|x_{n}-x\right\|^{2}=\phi(x),
\end{aligned}
$$

and hence $Q T x \in M$ because $Q T x \in C$. Furthermore, $M$ contains a fixed point of $Q T$. To this end, define

$$
M^{o}=\left\{v \in M:\|v-w\|=\min _{y \in M}\|w-y\|\right\} .
$$

Then, by Lemma $4, M^{o}$ is a singleton. Denote such a singleton by $z$. Then we have

$$
\|Q T z-w\|=\|Q T z-Q T w\| \leq\|z-w\|
$$

and hence $Q T z=z$. Applying the method of the proof of Theorem 1 to the nonexpansive mapping $Q T$, we obtain that $\left\{x_{t}\right\}$ converges strongly as $t \rightarrow 1$ to a fixed point $z$ of $Q T$. It remains to show that $z$ is a fixed point of $T$. Indeed, since $Q$ is sunny and nonexpansive retraction, from Lemma 2 , we get

$$
(T z-z, J(z-y)) \geq 0 \text { for all } y \in C .
$$

On the other hand, $T z$ belongs to $\operatorname{cl} I_{C}(z)$ by the weak inwardness condition. Hence for each integer $n \geq 1$, there exist $z_{n} \in C$ and $a_{n} \geq 0$ such that

$$
y_{n}:=z+a_{n}\left(z_{n}-z\right) \rightarrow T z \text { strongly. }
$$

Since $E$ has a uniformly Gâteaux differentiable norm, $J$ is uniformly continuous on bounded subsets of $E$ from the strong topology of $E$ to the weak-star topology of $E^{*}$. Thus it follows from (12) and (13) that

$$
\begin{aligned}
0 & \leq\left(T z-z, a_{n} J\left(z-z_{n}\right)\right) \\
& =\left(T z-z, J\left(a_{n}\left(z-z_{n}\right)\right)\right. \\
& =\left(T z-z, J\left(z-y_{n}\right)\right) \rightarrow(T z-z, J(z, T z))=-\|T z-z\|^{2} .
\end{aligned}
$$

Hence we have $T z=z$.

THEOREM 4. Let $E$ be a reflexive and strictly convex Banach space with a uniformly Gâteaux differentiable norm, $C$ a nonempty closed convex subset of $E$, and $T: C \rightarrow E$ a nonexpansive nonself-mapping satisfying the weak inwardness condition. Suppose that $C$ is a sunny nonexpansive retract of $E$, and that for some $u \in C$ and each $t \in(0,1), y_{t}$ is a (unique) fixed point of the contraction $R_{t}$ defined by (11), where $Q$ is a sunny nonexpansive retraction of $E$ onto $C$. If the fixed point set of $T$ is nonempty, then $\left\{y_{t}\right\}$ converges strongly as $t \rightarrow 1$ to a fixed point of $T$. 
Proof. The proof follows an idea of [22]. Let $x$ be a fixed point of $T$. Then we have

$$
\begin{aligned}
\left\|x-y_{t}\right\| & =\left\|Q x-Q\left(t T y_{t}+(1-t) u\right)\right\| \\
& \leq t\left\|x-T y_{t}\right\|+(1-t)\|x-u\| \\
& \leq t\left\|x-y_{t}\right\|+(1-t)\|x-u\|
\end{aligned}
$$

and hence $\left\|x-y_{t}\right\| \leq\|x-u\|$ for all $t \in(0,1)$. So $\left\{y_{t}\right\}$ is bounded. We now show that $\left\{y_{t}\right\}$ converges strongly as $t \rightarrow 1$ to a fixed point of $T$. To this end, let $t_{n} \rightarrow 1$ and $y_{n}=y_{t_{n}}$. As in proof of Theorem 1, define $\phi: C \rightarrow[0, \infty)$ by $\phi(z)=\mu_{n}\left\|y_{n}-z\right\|^{2}$ for each $z \in C$ and let

$$
M=\left\{u \in C: \mu_{n}\left\|y_{n}-u\right\|^{2}=\min _{y \in C} \mu_{n}\left\|y_{n}-y\right\|^{2}\right\} .
$$

Then $M$ is invariant under $Q T$. In fact, since $\left\{T y_{t}\right\}$ is bounded and

$$
\begin{aligned}
\left\|y_{t}-Q T y_{t}\right\| & \leq\left\|t T y_{t}+(1-t) u-T y_{t}\right\| \\
& =(1-t)\left\|u-T y_{t}\right\|,
\end{aligned}
$$

we have $y_{t}-Q T y_{t} \rightarrow 0$. So, we have for $z \in M$,

$$
\begin{aligned}
\left\|y_{n}-Q T z\right\| & \leq\left\|y_{n}-Q T y_{n}\right\|+\left\|Q T y_{n}-Q T z\right\| \\
& \leq\left\|y_{n}-z\right\|+\left\|y_{n}-Q T y_{n}\right\|
\end{aligned}
$$

and hence

$$
\mu_{n}\left\|y_{n}-Q T z\right\|^{2} \leq \mu_{n}\left\|y_{n}-z\right\|^{2}
$$

Then $Q T z \in M$ because $Q T z \in C$. Furthermore, by the proof of Theorem 3, we know that $M$ contains a fixed point of $Q T$, that is, there is a point $z$ such that $Q T z=z$. Since $Q$ is sunny and nonexpansive retraction, from Lemma 2, we have

$$
(T z-z, J(z-w)) \geq 0 \text { for all } w \in C .
$$

On the other hand, $T z$ belongs to $\mathrm{cl} I_{C}(z)$ by the weak inwardness condition. Hence for each integer $n \geq 1$, there exist $z_{n} \in C$ and $a_{n} \geq 0$ such that

$$
x_{n}:=z+a_{n}\left(z_{n}-z\right) \rightarrow T z \text { strongly. }
$$

As in the proof of Theorem 3, we have $T z=z$. For any $v \in F(T)$, we have

$$
t(v-u)+u=t v+(1-t) u=Q(t v+(1-t) u)
$$

and hence

$$
\left\|\left(y_{t}-u\right)+t(v-u)\right\|^{2}=\left\|Q\left(t T y_{t}+(1-t) u\right)-u-t(v-u)\right\|^{2}
$$




$$
\begin{aligned}
& \left.=\| Q\left(t T y_{y}-u\right)+u\right)-Q(t(v-u)+u) \|^{2} \\
& \leq\left\|t\left(T y_{t}-u\right)-t(v-u)\right\|^{2} \\
& \leq t^{2}\left\|y_{t}-v\right\|^{2} \\
& =t^{2}\left\|\left(y_{t}-u\right)-(v-u)\right\|^{2} .
\end{aligned}
$$

So, we have

$$
\begin{aligned}
0 & \geq\left\|\left(y_{t}-u\right)-t(v-u)\right\|^{2}-\left\|t\left(y_{t}-u\right)-t(v-u)\right\|^{2} \\
& \geq 2\left((1-t)\left(y_{t}-u\right), J\left(t\left(y_{t}-v\right)\right)\right) \\
& =2(1-t) t\left(y_{t}-u, J\left(y_{t}-v\right)\right)
\end{aligned}
$$

and hence

$$
\left(y_{t}-u, J\left(y_{t}-v\right)\right) \leq 0
$$

for $v \in F(T)$. From Lemma 1, it follows that

$$
\mu_{n}\left(x-z, J\left(y_{n}-z\right)\right) \leq 0
$$

for all $x \in C$. In particular, we have

$$
\mu_{n}\left(u-z, J\left(y_{n}-z\right)\right) \leq 0 .
$$

Combining (15) with $v=z$ and (16), we get

$$
\mu_{n}\left(y_{n}-z, J\left(y_{n}-z\right)\right)=\mu_{n}\left\|y_{n}-z\right\|^{2} \leq 0 .
$$

Therefore, there is a subsequence $\left\{y_{n_{1}}\right\}$ of $\left\{y_{n}\right\}$ which converges strongly to $z$. Suppose that there is another subsequence $\left\{y_{n_{k}}\right\}$ of $\left\{y_{n}\right\}$ which converges strongly to (say) $y$. Then $y$ is a fixed point of $Q T$ by (14) and also of $T$. Thus, as in the proof of Theorem 1, we have $z=y$ and hence $y_{t} \rightarrow z$.

Corollary 3. Let E, C, T, $Q$ be as in Theorem 3 (resp., Theorem 4). Suppose in addition that $C$ is bounded and that every weakly compact convex subset of $E$ has the FPP. Then for each $u \in C$, the sequence $\left\{x_{t}\right\}$ (resp., $\left.\left\{y_{t}\right\}\right)$ defined by (10) (resp., (11)) converges strongly as $t \rightarrow 1$ a fixed point of $T$.

Corollary 4 [27]. Let $H$ be a Hilbert space, $C$ a nonempty closed convex subset of $H, T: C \rightarrow H$ a nonexpansive nonself-mapping satisfying the weak inwardness condition, $P: H \rightarrow C$ the nearest point projection, and $\left\{x_{t}\right\}$ the sequence (resp., $\left.\left\{y_{t}\right\}\right)$ defined by (10) (resp., (11)) with $P$ instead of $Q$. If $T$ has a fixed point, then $\left\{x_{t}\right\}$ (resp., $\left.\left\{y_{t}\right\}\right)$ converges strongly as $t \rightarrow 1$ to a fixed point of $T$.

Proof. Note that the nearest point projection $P$ of a Hilbert space $H$ onto a closed convex subset $C$ is a sunny and nonexpansive retraction. Thus the result follows from Theorem 3 (resp., Theorem 4). 
Remark 3. Theorem 2, Theorem 3 and Theorem 4 apply to all uniformly convex and uniformly smooth Banach spaces and in particular, to all $L^{p}$ spaces, $1<p<\infty$.

Note added in proof. 1. Since $E$ is uniformly convex, the existence of the minimum in proofs of Lemma 3 and Theorem 2 also follows from [7, Proposition 2.2].

2. Since $\left\{x_{t}\right\}$ is a bounded approximating sequence and $E$ is uniformly convex, the existence of a fixed point of $T$ in proof of Theorem 2 also follows from Browder's demiclosedness principle [3].

3. The authors noticed, in the process of referring, the fact that Theorem 3 and 4 were proved in [22] with no assumption of strict convexity of $E$, using the stronger version of Theorem 1 for the self-mapping $T: C \rightarrow C$, where $C$ is a nonempty closed convex subset of $E$ which has normal structure.

Acknowledgement. The authors would like to thank Professor Hong Kun $\mathrm{Xu}$ for his kind help and giving them a copy of the paper [26] in press. They are also grateful to the anonymous referee for careful reading and helpful comments for the manuscript.

\section{REFERENCES}

[1] V Barbu and Th. Precupanu, Convexity and Optımization in Banach Spaces, Editura Academie1 R. S. R., Bucharest, 1978.

[2] F.E. BROWDER, Convergence of approximations to fixed points of nonexpansive mappings in Banach spaces, Arch. Rational Mech. Anal., 24 (1967), 82-90.

[3] F.E. BRowDER, Semicontractive and semiaccretive nonlinear mappings in Banach spaces, Bull. Amer. Math. Soc., 74 (1968), 660-665.

[4] D.F Cudia, The geometry of Banach spaces, smoothness, Trans. Amer. Math. Soc., 110 (1964), 284-314.

[5] M.M. DAY, Normed Linear Spaces, 3rd ed., Springer-Verlag, Berlin-New York, 1973.

[6] J. Diestel, Geometry of Banach Spaces, Lectures Notes in Math., 485, Springer Verlag, Berlin-Heidelberg, 1975.

[7] K. Goebel AND S. ReICH, Uniform Convexity, Hyperbolic Geometry and Nonexpansive Mappings, Marcel Dekker, New York-Basel, 1984.

[8] K.S. HA AND J.S. JUNG, On generators and nonlinear semigroups in Banach spaces, J. Korean Math. Soc., 25 (1988), 245-257

[9] K.S. HA AND J.S. JUNG, Strong convergence theorems for accretive operators in Banach spaces, J. Math. Anal. Appl., 147 (1990), 330-339.

[10] B. HALPERN, Fixed points of nonexpansive maps, Bull. Amer. Math. Soc., 73 (1967), 957961.

[11] J.S. JUNG AND S.S. KIM, Strong convergence theorems for nonexpansive nonself-mappings in Banach spaces, to appear in Nonlinear Anal.

[12] G.E. KIM AND W TAKAHASHI, Strong convergence theorems for nonexpansive nonself-mappings in Banach spaces, Nihonka1 Math. J., 7 (1996), 63-72.

[13] G. Marino AND G. TROMbetTa, On approxımatıng fixed points for nonexpansive maps, Indian J. Math., 34 (1992), 91-98. 
[14] S. ReICH, Asymptotic behavior of contractions in Banach spaces, J. Math. Anal. Appl., 44 (1973), 227-290.

[15] S. ReICH, Fixed points of condensing functions, J. Math. Anal. Appl., 41 (1973), 460-467

[16] S. REICH, An iterative procedure for constructing zeros of accretive sets in Banach spaces, Nonlinear Anal., 2 (1978), 85-92.

[17] S. ReICH, Product formulas, nonlinear semigroups and accretive operators, J. Funct. Anal., 36 (1980), 147-168.

[18] S. REICH, Strong convergence theorems for resolvents of accretive operators in Banach spaces, J. Math. Anal. Appl., 75 (1980), 287-292.

[19] S. REICH, On the asymptotic behavior of nonlinear semigroups and the range of accretive operators, J. Math. Anal. Appl., 79 (1981), 113-126.

[20] S. REICH, Convergence, resolvent consistency, and the fixed point property for nonexpansive mappings, Contemp. Math., 18 (1983), 167-174.

[21] S.P Singh AND B. Watson, On approxımatıng fixed points, Nonlinear Functional Analysis and 1ts Applications, Proc. Sympos. Pure Math., 45, part 2, Amer. Math. Soc., Providence, 1988, 393-395.

[22] W TAKaHASHI AND G.E. KIM, Strong convergence of approximants to fixed points of nonexpansive nonself-mappings in Banach space, Nonlinear Anal., 32 (1998), 447-454.

[23] W TAKAHASHI AND Y UEDA, On Reich's strong convergence theorems for resolvents of accretıve operators, J. Math. Anal. Appl., 104 (1984), 546-553.

[24] W TaKahashi and D.H. JeONG, Fixed point theorem for nonexpansive semigroups on Banach space, Proc. Amer. Math. Soc., 122 (1994), 1175-1179.

[25] H.K. XU, Inequalities in Banach spaces with applicatıons, Nonlinear Anal., 16 (1991), 11271138.

[26] H.K. XU, Approxımatıng curves of nonexpansive nonself mappings in Banach spaces, C. R. Acad. Sc1. Parıs Ser. I Math., 325 (1997), 179-184.

[27] H.K. XU AND X.M. YIN, Strong convergence theorems for nonexpansive nonself-mappings, Nonlinear Anal., 24 (1995), 223-228.

\author{
Department of Mathematics \\ DONG-A UNIVERSITY \\ PUSAN 604-714 \\ KOREA \\ E-mail: jungjs@seunghak.donga.ac.kr \\ Department of Applied Mathematics \\ Pukyong National University \\ PUSAN 608-737 \\ KOREA \\ E-mail: taehwa@dolphın.pknu.ac.kr
}

